\title{
Transmisión Intergeneracional del Apego y Función Reflexiva Materna: Una Revisión
}

\section{Intergenerational Transmission of Attachment and Reflective Function: A Revision}

\author{
Carolina Besoain * María Pía Santelices \\ Pontificia Universidad Católica de Chile
}

(Rec: 11 nov 2008 Acep.: 27 marzo 2009)

\begin{abstract}
Resumen
La investigación sobre la transmisión intergeneracional del apego ha asociado fuertemente los patrones de apego de los padres con los patrones de apego de infantiles. La evidencia ha mostrado que la sensibilidad de los padres es un importante precursor del apego seguro infantil, sin embargo, el mecanismo de transmisión a la base, sigue sin esclarecerse del todo. Algunos autores han propuesto además la función reflexiva materna como una de las variables explicativas. En este trabajo se expone una reflexión al respecto, mostrando ciertos hallazgos que muestran que la función reflexiva de la madre, y sus correlatos conductuales en la interacción madre-hijo, son un factor esencial en la transmisión intergeneracional del apego. Por último, se discute respecto a la importancia de incorporar estos hallazgos en el diseño de intervenciones preventivas.

Palabras clave: Apego infantil, apego adulto, transmisión intergeneracional del apego, función reflexiva, sensibilidad
\end{abstract}

\begin{abstract}
The research about intergenerational transmission of attachment has reported strong associations between the adult and child patterns of attachment. Evidence has shown parent's sensibility as an important precursor of infant's security. Nevertheless the transmission gap still isn't clear. Some authors have suggested the reflective function of the mother as a variable that could explain the gap. This article offers a discussion about this issue, showing some evidence that supports reflective function, and it's behavioral aspects in the mother-child early relationship, as an essential dimension in the explanation of the transmission. Finally we discuss the relevance of considering this evidence in the design of preventive interventions.
\end{abstract}

Key Words: Infant's attachment, adult's attachment, intergenerational transmission of attachment, reflective function, sensibility

\section{Introducción}

Las variables que explican el desarrollo del apego seguro, y especialmente la transmisión intergeneracional del apego, han sido una importante fuente de debate en la comunidad internacional. Particularmente, la pregunta por el mecanismo de transmisión, si bien ha encontrado algunas respuestas, sigue sin esclarecerse del todo.

Considerando la amplia evidencia que ha mostrado la fuerte asociación entre los patrones de apego materno y los patrones de apego de infantiles (Benoit \& Parker, 1994; Fonagy et al., 1991, Van Ijzendoorn, 1995), cobra relevancia estudiar con mayor detalle el mecanismo de transmisión, de modo de entender este fenómeno e intervenir adecuadamente, sobre todo en situación de repetición de apegos inseguros. Particularmente, la evidencia instala la pregunta sobre el vínculo que existiría entre el apego adulto o los modelos operativos internos de los padres, medidos con la Entrevista de Apego Adulto (AAI) (George et al., 1985) y las conductas de apego en la interacción con sus hijos mostradas en la Situación Extraña (Ainsworth, et al, 1978) a los 12 meses de edad. Al respecto, los resultados de Fonagy et al. (1991) fueron elocuentes; en el 75\% de los casos se pudo predecir con éxito si un infante iba a ser clasificado con un apego seguro o inseguro al año de edad a partir de la clasificación de apego de los padres.

En los últimos años se ha reportado la Función Reflexiva de los padres como un nuevo concepto que podría contribuir a entender el mecanismo que explica la transmisión del apego de padres a hijos, cuestionando la sensibilidad materna en el primer año de vida, como principal mediador del apego seguro infantil. Al respecto, el meta-análisis realizado por De Wolff \& Van Ijzendoorn (1997) plantea que la sensibilidad no puede ser considerada el factor exclusivo

Correspondencia a: msanteli@uc.cl; caritobesoain@gmail.com. 
o más importante en el desarrollo del apego. Asimismo, siembra dudas respecto a si la relación entre sensibilidad y seguridad es tan fuerte como mostraron los hallazgos iniciales de Ainsworth.

El presente trabajo explora algunos hallazgos que han surgido en la comunidad internacional respecto a los mecanismos causales y la transmisión intergeneracional del apego. Particularmente aquella evidencia que propone dimensiones asociadas a la función reflexiva o mentalización de la madre y sus correlatos conductuales en la interacción madre-hijo. A partir de ellos se realizará una reflexión respecto a la todavía poco clara relación entre los modelos operativos internos de la madre, abordados desde la AAI, y los patrones de apego desarrollados por sus hijos en la Situación Extraña. Específicamente se propondrá la función reflexiva materna como una alternativa explicativa que hace más parsimoniosa la unión entre apego adulto, modelos operativos internos de la madre y apego infantil.

\section{Algunos Conceptos Centrales}

\section{Apego Infantil}

La teoría del apego, creada por John Bowlby, enfatiza ciertas nociones teóricas clave respecto a los vínculos tempranos entre el bebé y sus cuidadores, siendo las más importantes que el bebé nace con una predisposición a vincularse a sus cuidadores, que éste organizará su comportamiento y pensamiento en función de la mantención de sus relaciones de apego, y que tenderá a mantener aquellas relaciones incluso si esto implica un alto costo de su propio funcionamiento (Slade, 1999). Estas formulaciones de Bowlby inspiraron la investigación de Ainsworth, cuya evidencia dio validación empírica a sus postulados (Slade, 1999). Uno de sus grandes aportes fue su distinción de tres grandes categorías de apego, seguro, ambivalente y evitativo, sobre la base de sus observaciones de los patrones infantiles de separación y reunión en el procedimiento de la Situación Extraña (Ainsworth, et al., 1978). Más tarde, Main y Solomon (1986) describieron una cuarta categoría insegura, el apego desorganizado.

Desde la teoría del apego, cuando se ha desarrollado un apego seguro, al infante le será posible explorar, sobre una base de seguridad y confianza, el mundo que lo rodea. Así podrá alcanzar las diversas tareas del desarrollo, llevando éste a su máximo potencial, si las condiciones de su entorno son favorables (Marvin \& Britner, 1999).

\section{Apego Adulto: Modelos Operativos Internos}

Para explicar la tendencia de los estilos de apego infantil a convertirse en una característica relacional o vincular del niño, posteriormente en el adulto, la teoría del apego recurre al concepto de modelo operante interno. Éste es definido por Bolwby (1988) como una representación del sí mismo, por una parte, y como una representación del sí mismo interactuando con una figura de apego en un contexto o entorno con carga emocional, por otra. Estos modelos influyen directamente en el modo en que un ser humano se siente con respecto a cada progenitor y con respecto a sí mismo, el modo en que espera ser tratado y el modo en que tratará a los demás cuando niño y posteriormente cuando adulto. Una vez construido, estos modelos tienden a persistir de manera más o menos estable a lo largo del tiempo y a operar a nivel inconsciente (Bowlby, 1988). Los modelos de los adultos se han clasificado en cuatro categorías: autónomos, preocupados, rechazantes y con trauma no resuelto (Dossier, Stovall \& Albus, 1999). Cada uno de estos estilos de apego de los padres, medidos a través de la AAI (George, et al, 1985), han demostrado una alta asociación con las conductas de apego infantil de sus hijos (Van Ijzendoorn, 1995).

\section{Sensibilidad del Cuidador}

Se define la respuesta sensible del cuidador como aquella conducta que éste realiza para responder a las demandas del bebé, incluyendo la capacidad de notar sus señales, poder interpretarlas adecuadamente y responder afectiva y conductualmente de manera apropiada y rápida (Bowlby, 1980, 1988, 1997, 2003). Los resultados de Aisworth y colaboradores (1971, citado en Slade, 1999) revelaron que los hijos de madres con mayores niveles de sensibilidad, tendían a mostrar un comportamiento de apego seguro en la Situación Extraña a los 12 meses de edad, instalando la centralidad de la sensibilidad materna en el primer año de vida como clave para el desarrollo de un apego seguro. Con posterioridad, otros investigadores han encontrado evidencia que apoya la relación entre sensibilidad materna y seguridad (eg. Isabella, 1993).

\section{Mentalización y Función Reflexiva del Cuidador}

Mentalizar es la capacidad de significar la experiencia de uno mismo y de los otros en términos de estados subjetivos y procesos mentales (Fonagy, et al, 2007). Esta capacidad se ha descrito como uno de los mayores logros del desarrollo, y se propone que alcanzarla es facilitado por relaciones de apego seguro. Es así como el entendimiento de sí mismo o de los otros, en términos de pensamientos, sentimientos y deseos, depende crucialmente de que se haya sido adecuadamente entendido por los propios cuidadores (Fonagy, et al, 2007, 2008). En este contexto la mentalización no es sólo un proceso cognitivo, sino un proceso en el cual la regulación afectiva tiene un rol fundamental, siendo ésta un preludio de la mentalización. Es así como una vez que la mentalización ha ocurrido, se transforma la naturaleza de la regulación afectiva, surgiendo la llamada afectividad mentalizada que consiste en 
una capacidad madura de regulación afectiva que permite descubrir los significados subjetivos de los propios estados afectivos (Fonagy et al., 2002).

En la misma línea, la función reflexiva refiere a la capacidad adulta de procesar experiencia intersubjetiva e interpersonal, siendo capaz de pensar sobre las emociones y su relación con el comportamiento (Fonagy, et al., 2002). Es una capacidad nuclear que permite a los padres acceder flexible y coherentemente a las emociones y memorias relevantes de las propias experiencias de apego tempranas, y asimismo, proveer una base segura a los propios hijos. Es posible entenderla como la capacidad de mentalizar respecto a las propias experiencias de apego tempranas.

\section{Nuevos Hallazgos: La Función Reflexiva como una alternativa para explicar la Transmisión Intergeneracional del Apego}

\section{Apego Adulto, Función Reflexiva y Apego Infantil.}

Con el objetivo de examinar la relación entre la función reflexiva materna, el apego adulto y el apego infantil, Slade y colaboradores (2005) administraron una serie de pruebas a 40 madres de clase media educada, de 31 años de edad promedio. A las 32 semanas de gestación, se les administró la Entrevista de Apego Adulto (George, et al., 1985). A los 10 meses desde el nacimiento de los bebés, se administró a las madres la Entrevista de Desarrollo Parental (PDI) (Aber et al, 1985, citado en Slade, et al, 2005), y a los 14 meses se administró a los bebés con sus madres la Situación Extraña (Ainsworth, et al, 1978).

Como primera hipótesis, se esperaba que la clasificación de apego materna (medida a partir de la AAI) estuviera asociada significativamente a la función reflexiva materna (medida a partir de la PDI). Los resultados mostraron que las madres con apego de tipo autónomo tenían una función reflexiva significativamente más alta que las madres con apego de tipo evitativo, preocupado y no resuelto, y que tanto las madres con apego de tipo evitativo como preocupado, tenían una función reflexiva más alta que las con apego de tipo irresuelto (Slade, et. al, 2005).

En una segunda hipótesis esperaba que el apego infantil fuera predicho por la función reflexiva materna. Los resultados revelaron poderosas relaciones entre la función reflexiva materna a los 10 meses del bebé, y la seguridad en el apego del bebé a los 14 meses. Específicamente, las madres de niños seguros tuvieron niveles significativamente más altos de función reflexiva que las madres de niños resistentes o desorganizados. Las madres de niños evitativos no tuvieron niveles de función reflexiva significativamente distintos que las madres de niños seguros (Slade, et. al, 2005).
En conjunto, los resultados de Slade et al (2005) indican que la capacidad reflexiva de la madre está relacionada no sólo a su estado mental en relación a su propio apego, sino también al apego de su hijo. Esto los lleva a preguntarse si acaso la ampliamente documentada correlación entre el apego adulto e infantil pudiese ser vista como un efecto indirecto de la función reflexiva parental, siendo esta variable la que esté directamente asociando a las otras dos, mediando su relación. Desde esta perspectiva, las categorías de la AAI estarían ofreciendo una manera de describir dimensiones de alta y baja función reflexiva, estando la seguridad asociada a una alta reflexividad y la evitación, preocupación y los estados no resueltos, asociados a variados tipos de fallas en la mentalización (Slade, et. al, 2005).

En otra publicación del mismo estudio, Grienenberger y colaboradores (2005) encontraron para la misma muestra una fuerte correlación negativa entre la ya descrita función reflexiva materna y las perturbaciones en la comunicación afectiva materna, medida con el Instrumento Atípico para Evaluación y Clasificación del Comportamiento Materno (AMBIANCE) de Lyons-Ruth y sus colaboradores (1999). A partir de ello, los autores proponen que las madres con mayor función reflexiva estarían mejor equipadas para manejar la vulnerabilidad emocional infantil, sin verse sobrepasadas por sus propios temores y hostilidad no integrados. Esto, en la medida que serían más hábiles en la distinción entre sus propios afectos y los afectos de sus hijos. Es así como proponen que la influencia de la función reflexiva materna sobre el apego infantil sería mediada por la capacidad de la madre de regular el temor y el distrés del bebé, sin asustarlo ni perturbarlo.

Es llamativo al respecto que las fallas maternas en la contención del afecto aparecen asociadas con más fuerza con el apego infantil que medidas previas de la sensibilidad materna (Grienenberger, et al, 2005).

\section{Mind-Mindedness, Sensibilidad y Comportamiento Materno}

Meins y colaboradores (2001) proponen que es preciso repensar el concepto de sensibilidad materna y el modo en que éste ha sido operacionalizado. Para ello utilizan el constructo de mind-mindedness, o mente mentalizante, acuñado por Meins (1997), que permitiría distinguir la sensibilidad general de la madre hacia las necesidades físicas y emocionales de sus hijos, de una sensibilidad más específica hacia los estados mentales del niño y su actividad consiguiente. Con ello buscan detenerse en la poco considerada distinción de Ainsworth entre la responsividad de las madres ante las claves emocionales de sus hijos y lo apropiado de esa respuesta. Específicamente evalúan, en una muestra de 71 madres y sus hijos de clase media-baja, el comportamiento materno en un contexto interaccional de juego con dos escalas, una de mind-mindedness materno creada por ellos y la escala de sensibilidad materna de Ainsworth, et al. 
(1971, citado en Meins et al., 2001). Su objetivo principal es investigar el poder relativo de cada uno de estos conceptos en la predicción del apego seguro.

Sus resultados arrojan, en primer lugar, que sensibilidad y mind-mindedness son constructos que miden aspectos relacionados, pero que son diferentes dimensiones de la interacción madre-hijo. En segundo lugar muestran que la dimensión de mind-mindedness que refiere a la realización de comentarios mentalísticos apropiados de la madre hacia el bebé, es la única dimensión que predice significativamente el apego seguro. Además, muestran que tanto la sensibilidad materna como los comentarios mentalísticos apropiados de la madre son predictores significativos del apego seguro. Sin embargo, esta última variable explica un monto de la varianza mucho mayor de lo que explica la sensibilidad $(12,7 \%$ y $6,5 \%$ respectivamente).

Lok \& McMahon (2006) examinan en 89 madres educadas de Sydney y sus primogénitos, las relaciones entre mind- mindedness (Meins 1999, citada en Lok \& McMahon, 2006) y una medida de interacción con un foco específico en la congruencia afectiva propuesto por Biriner et al (1998, citado en Lok \& McMahon, 2006) llamado disponibilidad emocional. Se entrevistó a las madres en sus casas cuando sus hijos tenían entre 4 y 5 años de edad. Además se videograbó a las madres y sus hijos en una interacción de juego inestructurada. Se hipotetizó que las madres que se enfocasen en los atributos mentalísticos de sus hijos, demostrando por tanto mayor mind- mindedness, estarían más disponibles emocionalmente en la interacción con sus hijos. Específicamente se esperaba que una mayor mindmindedness estuviera asociada fuertemente a la sensibilidad materna durante la interacción.

Los resultados respaldaron sólo parcialmente la hipótesis, mostrando que la mind- mindedness estaba significativamente asociada a la ausencia de hostilidad materna, tendiendo menos a ser hostiles en la interacción con sus hijos las madres con más mind- mindedness. Proponen que esta evidencia es consistente con la propuesta de Fonagy \& Target (1997, citado en Lok \& McMahon, 2006) respecto a que las respuestas autoritarias u hostiles al mal comportamiento podrían reflejar una menor capacidad de mentalización en los padres, y por tanto una menor facilitación para el desarrollo de mentalización en sus hijos. Sorpresivamente, no se encontró la asociación esperada entre mind- mindedness y sensibilidad materna. Al respecto, son relevantes las recomendaciones de Biringen, Brown, et al. (2000, citado en Lok \& McMahon, 2006), quienes notaron la necesidad de examinar las variadas dimensiones de la disponibilidad emocional separadamente, y sugieren que no sería adecuado tener un foco exclusivo en la sensibilidad materna, sin considerar otras variables de la interacción.

\section{Discusión}

La evidencia presentada en su conjunto permite algunas reflexiones respecto a la interrogante que rodea la relación entre los modelos operativos internos de la madre y el patrón de apego desarrollado por su hijo.

Fonagy ya en 1991 planteaba que el poder predictivo de la AAI residiría no en la cualidad de las experiencias pasadas de la madre, sino en la organización total de las estructuras mentales que subyacen las relaciones y los temas relacionados con el apego, siendo la madre de un niño con apego seguro capaz de comunicar fluidamente una representación global flexible sobre la relación con cada uno de sus padres durante su niñez. Sin embargo, todavía el proceso a través del que este Modelo Operativo Interno interactúa con el infante, y contribuye a la consolidación de un patrón de apego, no estaba claro en sus hallazgos.

Los resultados de Slade et al. (2005) de alguna manera confirman la intuición de Fonagy respecto a la capacidad reflexiva de la madre en la relación con su hijo como explicación para las diferencias en el repertorio interaccional que derivará en el establecimiento de apegos seguros o inseguros en los niños. La fuerte asociación entre la función reflexiva medida a través del PDI y los patrones de apego infantil al año de edad es elocuente. Asimismo, es un aporte en la medida que la función reflexiva es medida en un nuevo escenario, esto es, la relación actual entre la madre y su hijo, y no en relación a sus figuras de apego tempranas como hace la AAI. Es así como se avanza en la comprensión del proceso de transmisión, ya que confirma la intuición respecto a que dicha capacidad también estaría puesta en juego en la interacción temprana madre-hijo.

Los resultados de Grienenberger et al. (2005) contribuyen a precisar la relación entre función reflexiva y el comportamiento materno. La correlación negativa encontrada entre ésta y las perturbaciones en la comunicación afectiva materna, constituye evidencia significativa respecto a esclarecer el correlato conductual de la función reflexiva en la interacción madre-hijo. Ésta permitiría a la madre distinguir entre sus propios afectos y los afectos de su hijo, y por tanto la capacitaría mejor para manejar y regular los afectos negativos del bebé, manteniendo controlada su propia hostilidad y temor.

Por otro lado, es interesante la coherencia entre los resultados de Lok et al. (2006) respecto a la hostilidad materna y los resultados de Grienenberger sobre el manejo de afectos negativos. En ese sentido sería adecuado pensar que una mayor reflexividad estaría vinculada a un mejor manejo de los estados emocionales negativos del hijo, y por tanto a un mayor control de la propia hostilidad.

Tanto los hallazgos de Slade et al. (2005) y Grienenberger et al. (2005) de alguna manera contribuyen al entendimiento del planteamiento de De Wolff \& Van IJzendoorn (1997) respecto a que la sensibilidad materna no ha sido suficiente 
Figura 1. Transmisión Intergeneracional del Apego: Comparación entre Modelo basado en la Sensibilidad y otros Modelos basados en la Función Reflexiva.

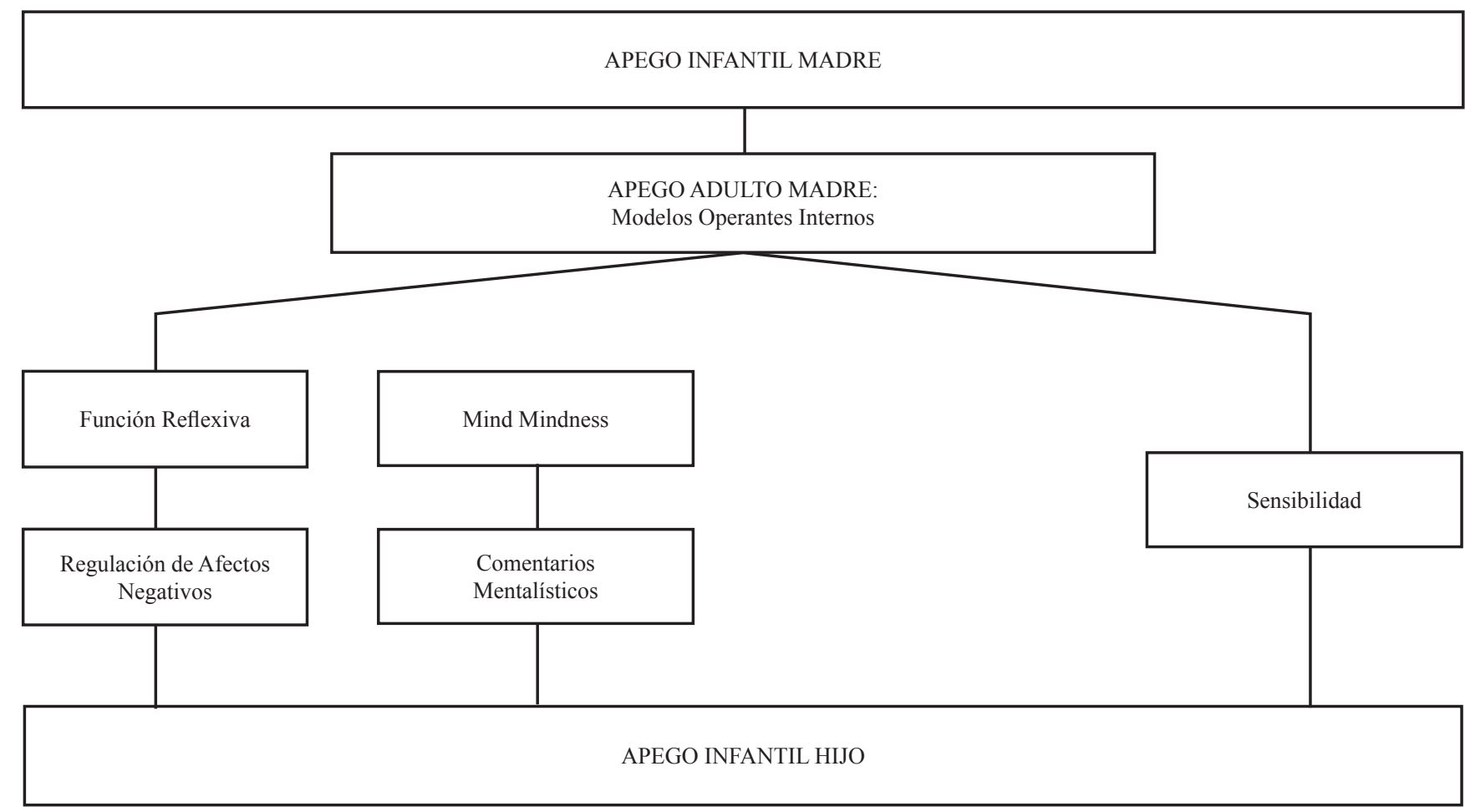

para explicar el desarrollo del apego infantil. La propuesta de Slade et al. (2005) al respecto es que los programas de intervención debieran centrarse principalmente en el desarrollo de la función reflexiva en las madres, incluso con mayor énfasis que en el desarrollo de habilidades parentales. En sus palabras, es necesario ayudarles a las madres a pensar sobre su conducta, más que cambiar su conducta, sin desestimar que los cambios en el comportamiento pueden ser seguidos por cambios en el pensamiento y la representación. Si se entiende la función reflexiva como el principal mecanismo de transmisión del apego, es posible entender que los esfuerzos para modificar el comportamiento parental centrados en el desarrollo de habilidades parentales hayan mostrado baja efectividad (Slade, et al, 2005), y que se haya encontrado solo asociaciones moderadas entre el comportamiento materno y el apego infantil (Van Ijzendoorn, 1995).

Los hallazgos de Meins et al. (2001) en la misma línea, plantean la necesidad de redefinir el concepto de sensibilidad y su operacionalización, denunciando la falta de precisión de los estudios al respecto de la ocurrencia de la responsividad y la diferencia con lo apropiada que ésta pueda ser. Sus hallazgos, al igual que los de Grienenberger et al (2005), contribuyen a entender el correlato comportamental de la función reflexiva en la interacción madre-hijo, introduciendo la importancia de la realización de comentarios mentalísticos apropiados de la madre hacia el bebé, en el desarrollo del apego seguro. En este escenario el constructo de mind-mindedness materno se plantea como una buena alternativa para la redefinición de la sensibilidad, en la medida que considera la propiedad de las interacciones de la madre con su hijo, y no sólo su ocurrencia. Asimismo, incorpora el nivel representacional de la sensibilidad, repensándola en términos de la tendencia específica a enfocarse y responder a los estados mentales de sus hijos.

Tal como Meins et al. (2001) plantea, esta variación constituye un aporte fundamental, en la medida que el constructo de mind-mindedness, a diferencia de la sensibilidad, al introducir el componente representacional, posibilita una asociación teórica con el concepto de Modelos Operativos Internos. Si el desarrollo del apego seguro infantil depende de las estructuras mentales que subyacen las relaciones y los asuntos relacionados con la experiencia de apego temprano de la madre, tal como se ha propuesto teóricamente y se ha demostrado empíricamente al asociar la AAI con los resultados de la Situación Extraña al año de edad, la evidencia presentada es un aporte en cuanto a construir una teoría coherente y con los planteamientos iniciales de John Bowlby. Esta evidencia muestra que sería la misma función reflexiva de la madre medida en la AAI, la que se despliega en la interacción con su hijo, traduciéndose directamente, a través de los comentarios mentalísticos apropiados, o indirectamente, a través de la regulación adecuada de estados emocionales negativos, y causando finalmente el establecimiento de 
un patrón de apego particular. Con ello, tal como propone Meins et al. (2001) se hace más parsimoniosa la unión entre la Teoría del Apego y de los Modelos Operativos Internos con la evidencia empírica respecto a sus precursores y su transmisión intergeneracional (ver figura 1).

Sin embargo, es importante considerar que el fenómeno de la transmisión intergeneracional es de una alta complejidad. No sería razonable esperar que ésta fuese explicada en su totalidad por una sola dimensión, sea ésta la sensibilidad o bien la función reflexiva. Mucho más probable, y así lo sugiere la evidencia hasta la fecha, es que la transmisión esté afectada por múltiples dimensiones, y que cada una de ellas explique cierto monto de la varianza. Ahora bien, los nuevos hallazgos evidencian la necesidad de revisar la hegemonía de la sensibilidad materna como precursor del apego, sobre todo cuando se trata de diseñar intervenciones clínicas y de promoción del apego seguro. Olds y colaboradores (2007) destacan la importancia de considerar la trascendencia del uso de la teoría, los datos epidemiológicos y la evidencia empírica disponible para identificar aquellos aspectos que son relevantes en el ejercicio de la parentalidad. En este llamado, la consideración de la función reflexiva es prioritaria, tanto en vista de la evidencia empírica discutida en este trabajo como de los postulados teóricos de John Bowlby y otros autores que han continuado su legado.

\section{Referencias}

Ainsworth, M. D. S., Blehar, M. C., Waters, E. \& Wall, S. (1978). Patterns of attachment: A psychological study of the strange situation. Hillsdale, NJ: Erlbaum.

Benoit, D. \& Parker, K. (1994). Stability and transmission of attachment across three generations. Child Development, 65, 1444 - 1457.

Bowlby (1980) La Pérdida Afectiva. Buenos Aires: Paidós.

Bowlby (1988) A Secure Base: Clinical applications of attachment theory. London: Routledge.

Bowlby (1997). El Vínculo Afectivo. España: Paidós

Bowlby (2003). Vinculos Afectivos: Formación, Desarrollo y Pérdida. Madrid: Morata.
De Wolff, M. S. \& Van IJzendoorn, M. H. (1997). Sensitivity and attachment: A meta-analysis on parental antecedents of infant attachment. Child Development, 68, 571- 591.

Dozier, M., Stovall, K., \& Albus, K. (1999). Attachment and psychopathology in adulthood. En: J. Cassidy \& P. Shaver (eds.) Handbook of Attachment: Theory, Research, and Clinical Applications (pp. 497- 519). New York: The Guilford Press.

Fonagy, P., Steele, H. \& Stelle, M.(1991). Maternal representations of attachment during pregnancy predict the organization of infant- mother attachment at one year of age. Child Development, 62, 891- 905.

Fonagy, P., Gergely, G., Jurist, E. Target, M. (2002). Affect Regulation, Mentalization, and the Development of the Self. New York: Other Press.

Fonagy, P., Bateman, A. (2007). Mentalizing and borderline personality disorder. Journal of Mental Health, 16, 83-101.

George, C., Kaplan, M., \& Main, M. (1985). Adult attachment interview. Unpublished manuscript, University of California, Berkeley.

Isabella, R. A. (1993). Origins of attachment: Maternal interactive behavior across the first year. Child Development, 64, 605-621.

Lok, S. \& McMahon, C. (2006). Mother's thougts about their children. Links between mind- mindedness and emotional availability. British Journal of Develoment Psychology, 24, 477- 488.

Main, M. \& Solomon, J. (1986) Discovery of a new, insecure-disorganized/ disoriented attachment pattern. En T. B. Brazelton \& M. Yogman (eds.). Affective development in infancy (pp. 95-124). Norwood, NJ: Ablex.

Marvin, R. \& Britner, P. (1999) Normative development: The ontogeny of attachment. En J. Cassidy \& P. Shaver (eds.) Handbook of Attachment: Theory, Research, and Clinical Applications (pp. 44- 67). New York: The Guilford Press.

Meins, E., Fernyhough, C., Fradley, E., Tuckey, M. (2001) Rethinking maternal sensitivity: Mothers' comments on infants' mental processes predict security of attachment at 12 months. J. Child Psychological. Psychiatrics, 42, 637- 648 .

Slade, A. (1999) Attachment theory and research: Implications for the theory and practice of individual psychotherapy with adults. En J. Cassidy \& P. Shaver (eds.) Handbook of Attachment: Theory, Research, and Clinical Applications (pp. 575- 594). New York: The Guilford Press.

Slade, A., Grienenberger, J., Bernbach, D. \& Locker, A. (2005). Maternal reflective functioning, attachment, and the transmission gap: A preliminary study. Attachment \& Human Development, 7, 283- 298.

Van Ijzendoorn, M. (1995) Adult attachment representations, parental responsiveness, and infant attachment: A meta-analysis on the predictive validity of the adult attachment interview. Psychological Bulletin, $117,387-403$. 